\title{
1D Confocal Broad Area Semiconductor Lasers (Confocal BALs) for Fundamental Transverse Mode Selection (TMS\#0)
}

\author{
Henning Fouckhardt $\mathbb{D}$, Ann-Kathrin Kleinschmidt, \\ Johannes Strassner $(\mathbb{D}$, and Christoph Doering $(\mathbb{D})$ \\ Integrated Optoelectronics and Microoptics Research Group, Physics Department, Technische Universität Kaiserslautern (TUK), \\ P.O. Box 3049, D-67653 Kaiserslautern, Germany \\ Correspondence should be addressed to Henning Fouckhardt; fouckhar@physik.uni-kl.de
}

Received 11 March 2019; Accepted 16 April 2019; Published 17 June 2019

Academic Editor: Vasily Spirin

Copyright (C) 2019 Henning Fouckhardt et al. This is an open access article distributed under the Creative Commons Attribution License, which permits unrestricted use, distribution, and reproduction in any medium, provided the original work is properly cited.

\begin{abstract}
Previously in this journal we have reported on fundamental transverse mode selection (TMS\#0) of broad area semiconductor lasers (BALs) with integrated twice-retracted $4 f$ set-up and film-waveguide lens as the Fourier-transform element. Now we choose and report on a simpler approach for BAL-TMS\#0, i.e., the use of a stable confocal longitudinal BAL resonator of length $L$ with a transverse constriction. The absolute value of the radius $R$ of curvature of both mirror-facets convex in one dimension (1D) is $R=L$ $=2 f$ with focal length $f$. The round trip length $2 L=4 f$ again makes up for a Fourier-optical $4 f$ set-up and the constriction resulting in a resonator-internal beam waist stands for a Fourier-optical low-pass spatial frequency filter. Good TMS\#0 is achieved, as long as the constriction is tight enough, but filamentation is not completely suppressed.
\end{abstract}

\section{Introduction}

Broad area (semiconductor diode) lasers (BALs) are intended to emit high optical output powers (where "high" is relative and depending on the material system). As compared to conventional narrow stripe lasers, the higher power is distributed over a larger transverse cross-section, thus avoiding catastrophic optical mirror damage (COMD). Typical BALs have emitter widths of around $100 \mu \mathrm{m}$.

The drawback is the distribution of the high output power over a large number of transverse modes (in cases without countermeasures) limiting the portion of the light power in the fundamental transverse mode (mode \#0), which ought to be maximized for the sake of good light focusability.

Thus techniques have to be used to support, prefer, or select the fundamental transverse mode (transverse mode selection TMS\#0) by suppression of higher order modes already upon build-up of the laser oscillation.

In many cases reported in the literature, either a BAL facet, the transverse effective refractive index distribution, or the pump current distribution is modified [1-8]. Or an external cavity is employed [7-14]. In all these instances eventually low-pass spatial frequency filtering is performed. Since feedback from an external cavity may also cause selfpulsation due to destabilization of the emission process [1519], the transverse mode selection set-up might also be integrated into the laser resonator $[20,21]$, a concept which we presented earlier. Moreover, approaches with tapered lasers or amplifiers or similar devices are known [22-25].

Previously in this journal we have also reported on a concept for TMS\#0, which has employed a twice-retracted integrated $4 f$ set-up with an actual length of $1 f$ forming the laser resonator [26]. One facet has incorporated the spatial frequency filter, while the other one has housed a film-waveguide lens as the 1D Fourier-transform element. Experimental results have shown good TMS\#0. The best one-dimensional beam quality parameter measured has been $M_{1 \mathrm{D}}^{2}=1.47$.

A technological disadvantage of the latter approach has been the sophisticated preparation of the film-waveguide lens with a necessary dry-etch depth precision better than (i.e., below) $20 \mathrm{~nm}$. Here we propose a simpler resonator design. 


\section{Concept and Laser Design}

In this contribution, we propose and report on the realization of a confocal BAL resonator with (in top-view) a bowtie-shaped beam constriction of minimal width $a$ defining the smallest transverse beam width half-way between the cylindrical facets with Fresnel reflection. These mirror-facets are both convex in 1D (viewed from outside the resonator), giving a stable resonator.

Typically confocal resonators are not employed for semiconductor lasers. An early contribution with a so-called confocal resonator is given in [27]. But one of the mirrorfacets had been convex, while the other one had been concave or plane, yielding an unstable resonator. In our case, only mirror-facets, which are convex in 1D (see above) and of equal absolute value for the radius of curvature, are employed.

A confocal resonator is defined by the following equation:

$$
R=L=2 f
$$

where $R$ is the absolute value of the radius of curvature of both facets, $L$ the resonator length, $2 L$ the round trip length, and $f$ the common (absolute value of the) focal length of the curved mirror-facets.

Both 1D curved mirror-facets perform a 1D spatial Fourier-transform, each from their respective front to their back focal plane. Since the resonator length is $2 f$, these focal planes coincide with the plane in the longitudinal middle of the resonator, called the "middle plane" from now on.

Rays with low propagation angles with respect to the optical axis account for low spatial frequencies and thus for the fundamental transverse mode $(\# 0)$. They are Fresnelreflected back into the resonator at the cylindrical mirrorfacets with a reflectivity of about 31\%. Rays with larger propagation angles, which correlate with larger spatial frequencies and higher transverse modes, are blocked by the transverse constriction, the latter thus acting as a 1D low-pass spatial frequency filter, intended to support the fundamental transverse mode.

Figure 1 contains a light microscope image of one of our confocal BAL resonators in top-view with a bow-tie-shaped, dry-etched laser ridge and a constriction with a width of (in this case) $32 \mu \mathrm{m}$ in the middle plane [28]. The absolute value of the radius of curvature is $R=1 \mathrm{~mm}$ for both convex facets, identical to the resonator length $L$. To the best of our knowledge, the spatial resolution of the etch process does not affect the symmetry of the bow-tie shape.

The advantage of using a confocal resonator for Fourieroptical spatial frequency filtering is its ease of design and technological preparation as well as the fact that a relatively wide opening angle of the mirror-facets can be used even for a tide constriction $a$ (beam width on facet $b>a$ ). That is, the transverse beam width $b$ on the facets is considerably larger than the width $a$ of the constriction (spatial frequency filter) in the middle plane. The latter aspect is schematically illustrated in Figure 2.

The layer sequence of our lasers is based on the AlGaAsSb material system on GaAs substrates. The devices are pn junctions, i.e., laser diodes, edge-emitting with a $450 \mathrm{~nm}$ thick active region consisting of eight Stransky-Krastanov-grown

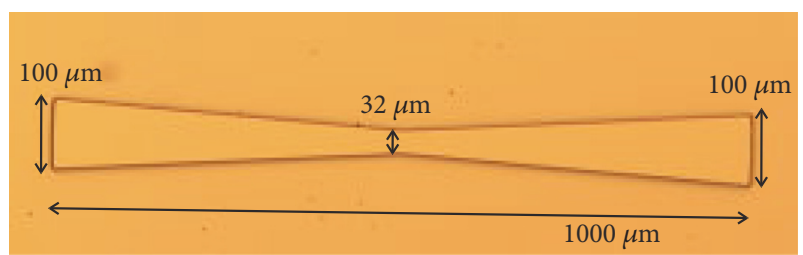

FIGURE 1: Light microscope image (top-view) of a confocal BAL resonator with a bow-tie-shaped laser ridge and, in this example, a $32 \mu \mathrm{m}$ wide constriction in the middle plane. The resonator length and the radii of curvature are $1 \mathrm{~mm}$.

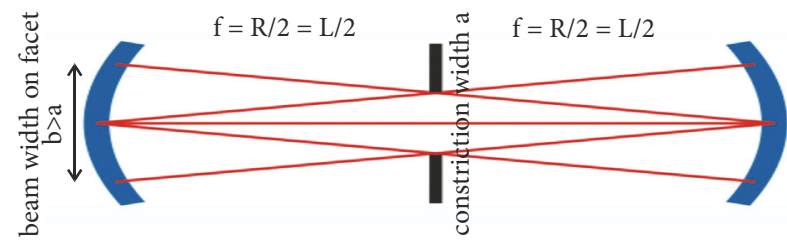

FIGURE 2: Principal sketch of the confocal resonator with constriction. The transverse beam width $b$ on the facets is considerably larger than the opening width $a$ of the low-pass spatial frequency filter in the middle plane.

GaAsSb quantum dot (QD) layers in-between $50 \mathrm{~nm}$ wide GaAs barriers [28]. The lasers emit at wavelengths of ca. 930 nm.

It has to be stressed here that our confocal BAL approach is not restricted to this material system and laser design, but it rather represents a general concept. Even unipolar quantum cascade lasers for the $\mathrm{THz}$ emission range might profit from it.

For comparison, we prepared several lasers from the same batch/wafer: with no constriction or constrictions of 64,48 , 32 , and $16 \mu \mathrm{m}$ as the smallest transverse width, respectively. In all cases, the bow-ties were $100 \mu \mathrm{m}$ wide at the outer edges (see Figure 1 again). The device with a $16 \mu \mathrm{m}$ wide constriction did not oscillate/lase.

\section{Experimental Results and Discussion}

Figure 3 gives the laser characteristics for the confocal BAL, e.g., with the $64 \mu \mathrm{m}$ wide constriction (low-pass spatial frequency filter) to verify that the laser devices are of very good quality, even at continuous wave and room temperature operation. The differential quantum efficiency is $31.5 \%$ here.

For later comparison, Figure 4 reveals the results in terms of the near- and far-field intensity distributions for the confocal BAL without any constriction. The device operating temperature has been around $90 \mathrm{~K}$.

And Figure 5 gives false-color plots (intensity coded as colors, black/blue for low intensities, white/red/yellow for large intensities) of the near-field transverse intensity distributions for confocal BALs of the same batch with different smallest widths of the constriction. Actually the uppermost plot is from the confocal BAL without constriction, and the other ones stem from the devices with 64,48 , and $32 \mu \mathrm{m}$ wide (smallest) constriction, respectively. (Please remember: 


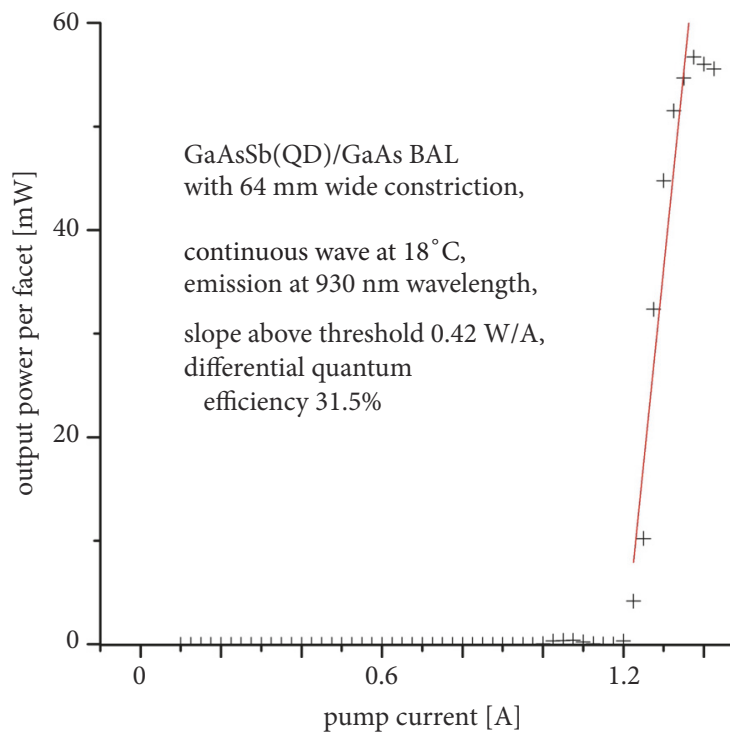

FIGURE 3: Laser characteristics of a confocal BAL with $64 \mu \mathrm{m}$ wide constriction just to show that the lasers are of very good quality. The laser threshold is at $1.2 \mathrm{~A}$, and the output power per facet shows values around $60 \mathrm{~mW}$ for a pump current of about $16 \%$ above threshold.
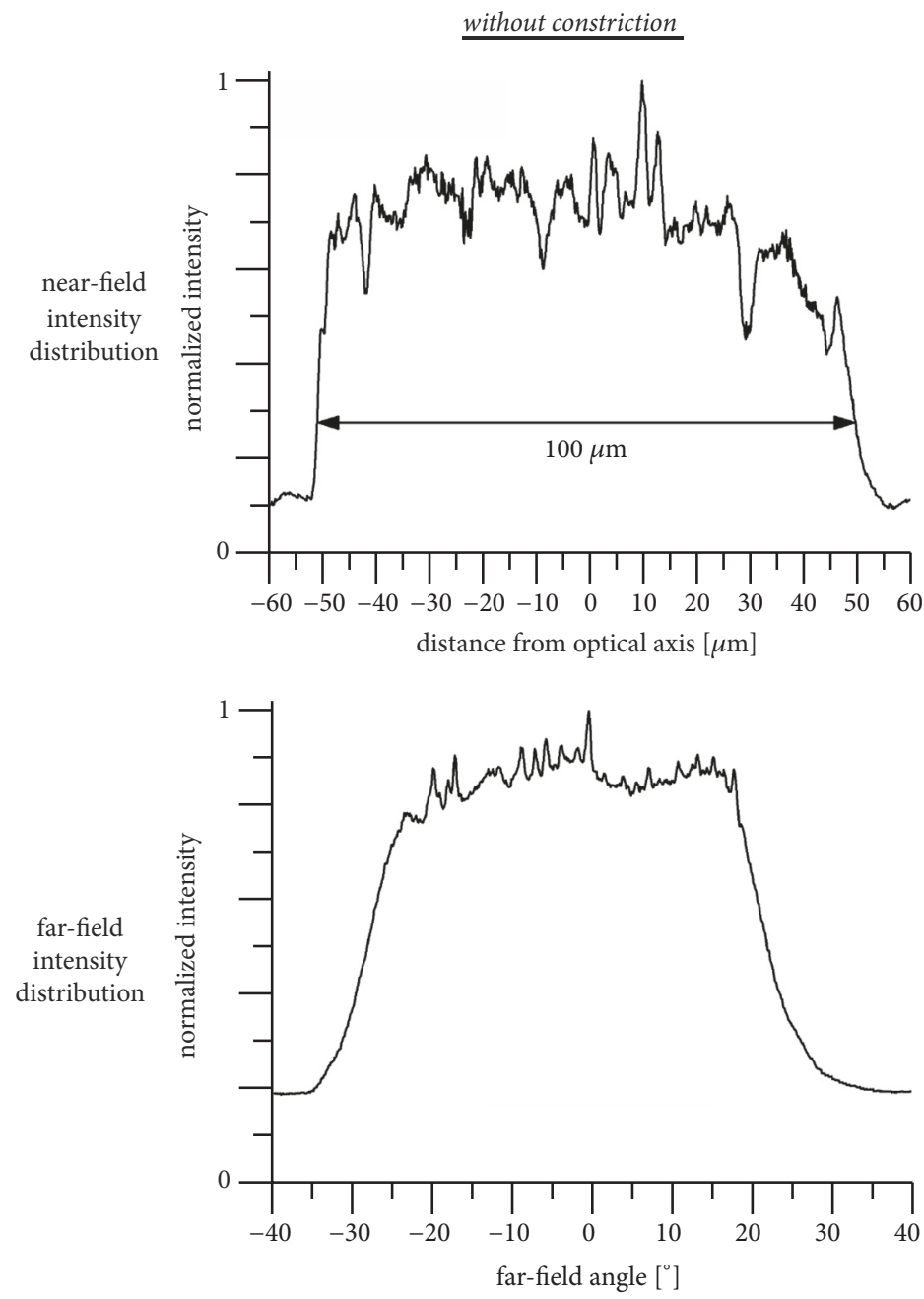

FIgURE 4: Near- and far-field intensity distributions for the confocal BAL without constriction, for comparison. The laser has been operated around $90 \mathrm{~K}$ in continuous-wave emission. 


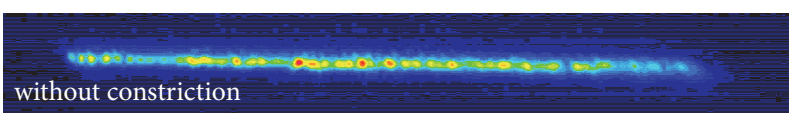

(a)

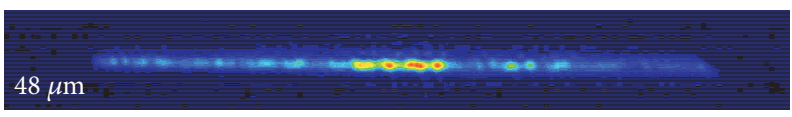

(c)

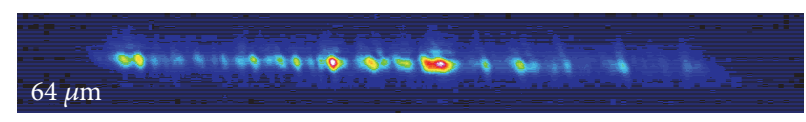

(b)

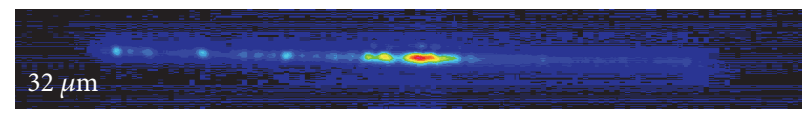

(d)

FIGURE 5: False-color plots of the near-field transverse intensity distributions for confocal BALs of the same batch with different widths of the constriction in the middle plane (i.e., no constriction, 64,48 , and $32 \mu \mathrm{m}$ wide, respectively). In all cases, the pump current has been around $16 \%$ above laser threshold and the lasers have been operated around $90 \mathrm{~K}$ in continuous-wave emission.

the device with a $16 \mu \mathrm{m}$ narrow constriction did not lase!) In all cases the pump current has been around $16 \%$ above laser threshold. Again the device operating temperature has been around $90 \mathrm{~K}$.

In the sequence of results in Figure 5, an increasingly stronger confinement of the transverse intensity distribution is obvious, resulting in a near-field intensity distribution similar to that of the desired fundamental transverse mode for a constriction with a width of $32 \mu \mathrm{m}$ the middle plane. But filamentation is not completely suppressed.

To make a closer inspection possible, Figure 6 (top and middle row) shows both the near- and the far-field intensity distribution for the same operating conditions for the confocal BAL with $32 \mu \mathrm{m}$ wide constriction in the middle plane. Gaussian fits (red lines) are added as guides to the eye.

Obviously the fundamental transverse mode is supported, but filamentation is not totally suppressed.

Both the near- and the far-field intensity distributionsmeasured with the help of objective lenses- show an intensity offset. As illustrated in the bottom part of Figure 6 by a sideview sketch and a top-view scanning electron micrograph (SEM), this is due to the fact that the mirror-facets have been dry-etched, resulting in some distance (ca. $36 \mu \mathrm{m}$ long) between the bow-tie edge and the device/crystal edge. Within this distance, the substrate has been laid bare upon etching, giving a plateau with a roughened surface. Part of the emitted light is diffusely reflected or rather scattered off the plateau. The scattered intensity portion accounts for the offset.

At pump currents, more than $20 \%$ above threshold considerable TMS\#0 is not observed, a problem which our approach has in common with most TMS concepts.

Comparing devices (from the same wafer) we have not found any significant deviations in differential quantum efficiency within our device and measurement tolerances for different smallest widths of the constriction (except for the fact that the device with $16 \mu \mathrm{m}$ constriction did not lase/oscillate at all). But the devices without any constriction had a worse quantum efficiency, worse by up to a factor of 10 . This is an unexpected result, since the TMS\#0 via a confocal resonator with constriction should increase the fraction of the total power in the fundamental transverse mode, but not necessarily the overall power. Further investigations have to be pursued on this issue.
As can be seen from the figure captions, the devices have been operated (continuous wave) both at around $90 \mathrm{~K}$ and at room-temperature $\left(18^{\circ} \mathrm{C}\right)$. In both cases, we did not observe a significant increase in device instability upon a temperature change by a couple of ten degrees Celsius.

The (even in the cases with constriction) still strong filamentation is also an unexpected result, since the bow-tieshape of the confocal resonator should have restricted the possible longitudinal paths for gain filaments geometrically. On the other hand, light scattering from the roughness of the etched transverse bow-tie edges might cause an additional coupling of the gain filaments. Thus an attempt to improve the TMS\#0 (i.e., to reduce the filamentation) further should go for a reduction of the mentioned roughness, which has been on the order of 100 to $500 \mathrm{~nm}$ (rootmean-square nominally) so far due to the roughness of the structures on the lithographic mask as well as the roughness induced by the reactive ion etching process itself.

In the case with the $32 \mu \mathrm{m}$ wide constriction in the middle plane, the intensity distributions in Figures 5(d) and 6 with a single-lobed far-field and a full far-field angle of $5.1^{\circ}$ (disregarding filamentation for a moment) allow for the extraction of a 1D beam quality parameter of $M_{1 D}^{2}=1.71$.

\section{Conclusions}

A concept for fundamental spatial transverse mode selection (TMS\#0) of edge-emitting broad area (semiconductor diode) lasers (BALs) is presented, which employs a $1 \mathrm{D}$ confocal resonator with a constriction in the middle plane, i.e., the plane half-way between the equally strongly curved convex mirror-facets. This plane serves both as the front and the back focal plane of the curved facets and, thus, also as the Fouriertransform plane in the sense of a Fourier-optical $4 f$ set-up. A transverse constriction in this plane is employed as a lowpass spatial frequency filter in order to select the fundamental transverse mode (TMS\#0).

Several lasers have been prepared from the same batch, differing from one another in the smallest width of the transverse constriction. The lasers are of very good quality, revealed by a differential quantum efficiency of around $30 \%$. 

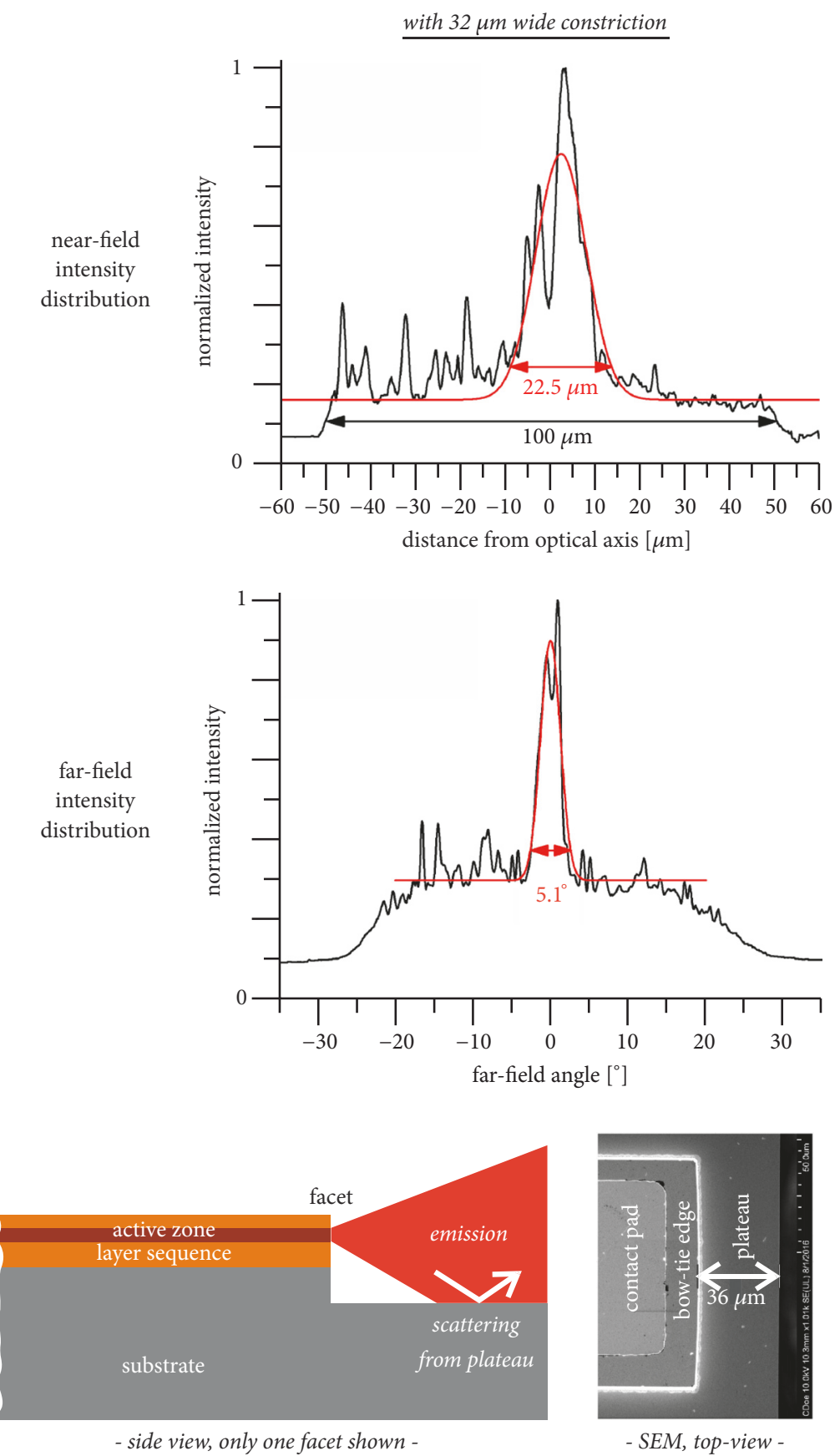

FIgURE 6: Top and middle row: near- and far-field intensity distributions for the confocal BAL with a $32 \mu \mathrm{m}$ wide constriction in the middle plane. Gaussian fits (red lines) are added for both intensity distributions as guides to the eye. The laser has been operated around $90 \mathrm{~K}$ in continuous-wave emission. Bottom: side-view sketch and top-view scanning electron micrograph (SEM) of one of the dry-etched mirrorfacets. The radiation is partially diffusely reflected or scattered off the somewhat rough substrate plateau, which has resulted from the dry-etch process to define the mirror-facets.

Transverse mode selection (TMS\#0) is indeed achieved via the confocal resonator design, that is for pump currents not larger than $20 \%$ above threshold. Filamentation is not completely suppressed.

The best TMS\#0 is achieved in case of the $32 \mu \mathrm{m}$ wide constriction. Here the measured one-dimensional (1D) beam quality parameter is $M^{2}{ }_{1 \mathrm{D}}=1.71$.

\section{Data Availability}

Most of the experimental data used to support the findings of this study are included within the article. Partially previously reported studies and experimental data were used to support this study. These prior studies and datasets are cited at relevant places within the text as references. Further or more 
detailed data used to support the findings of this study are available from the corresponding author upon request.

\section{Conflicts of Interest}

The authors declare that there are no conflicts of interest regarding the publication of this paper, neither concerning the funding nor for any other reason.

\section{Acknowledgments}

This research has been funded by the German Research Foundation (Deutsche Forschungsgemeinschaft, DFG) under contracts FO157/44 and FO157/46. Technological assistance by the Nano Structuring Center (NSC) of the Technische Universität Kaiserslautern (TUK) is gratefully acknowledged.

\section{References}

[1] K. Shigihara, Y. Nagai, S. Kakimoto, and K. Ikeda, "Achieving broad-area laser diodes with high output power and singlelobed far-field patterns in the lateral direction by loading a modal reflector," IEEE Journal of Quantum Electronics, vol. 30, no. 8, pp. 1683-1690, 1994.

[2] J. P. Hohimer, G. R. Hadley, and A. Owyoung, "Mode control in broad-area diode lasers by thermally induced lateral index tailoring," Applied Physics Letters, vol. 52, no. 4, pp. 260-262, 1988.

[3] H. Wenzel, P. Crump, J. Fricke, P. Ressel, and G. Erbert, "Suppression of higher-order lateral modes in broad-area diode lasers by resonant anti-guiding," IEEE Journal of Quantum Electronics, vol. 49, no. 12, pp. 1102-1108, 2013.

[4] J. P. Leidner and J. R. Marciante, "Beam quality improvement in broad-area semiconductor lasers via evanescent spatial filtering," IEEE Journal of Quantum Electronics, vol. 48, no. 10, pp. 1269-1274, 2012.

[5] M. Winterfeldt, P. Crump, S. Knigge, A. Maaßdorf, U. Zeimer, and G. Erbert, "High beam quality in broad area lasers via suppression of lateral carrier accumulation," IEEE Photonics Technology Letters, vol. 27, no. 17, pp. 1809-1812, 2015.

[6] J. Rong, E. Xing, Y. Zhang et al., "Low lateral divergence 2 $\mu \mathrm{m} \mathrm{InGaSb/AlGaAsSb}$ broad-area quantum well lasers," Optics Express, vol. 24, no. 7, pp. 7246-7252, 2016.

[7] C. Zink, M. Niebuhr, A. Jechow, A. Heuer, and R. Menzel, "Broad area diode laser with on-chip transverse Bragg grating stabilized in an off-axis external cavity," Optics Express, vol. 22, no. 12, pp. 14108-14113, 2014.

[8] M. Niebuhr, C. Zink, A. Jechow, A. Heuer, L. B. Glebov, and R. Menzel, "Mode stabilization of a laterally structured broad area diode laser using an external volume Bragg grating," Optics Express, vol. 23, no. 9, pp. 12394-12400, 2015.

[9] C. Simmendinger, D. Preißer, and O. Hess, "Stabilization of chaotic spatiotemporal filamentation in large broad area lasers by spatially structured optical feedback," Optics Express, vol. 5, no. 3, pp. 48-54, 1999.

[10] N. McCarthy, S. Mailhot, and Y. Champagne, "Single-mode operation of a broad-area semiconductor laser with an anamorphic external cavity: Experimental and numerical results," Applied Optics, vol. 39, no. 7-36, pp. 6806-6813, 2000.
[11] S. Wolff and H. Fouckhardt, "Intracavity stabilization of broad area lasers by structured delayed optical feedback," Optics Express, vol. 7, no. 6, pp. 222-227, 2000.

[12] V. Raab and R. Menzel, "External resonator design for highpower laser diodes that yields $400 \mathrm{~mW}$ of $\mathrm{TEM}_{00}$ power," Optics Express, vol. 27, no. 3, pp. 167-169, 2002.

[13] S. K. Mandre, I. Fischer, and W. Elsässer, "Control of the spatiotemporal emission of a broad-area semiconductor laser by spatially filtered feedback," Optics Express, vol. 28, no. 13, pp. 1135-1137, 2003.

[14] S. Wolff, A. Rodionov, V. E. Sherstobitov, and H. Fouckhardt, "Fourier-optical transverse mode selection in external-cavity broad-area lasers: experimental and numerical results," IEEE Journal of Quantum Electronics, vol. 39, no. 3, pp. 448-458, 2003.

[15] T. Heil, I. Fischer, and W. Elsäßer, "Coexistence of lowfrequency fluctuations and stable emission on a single high-gain mode in semiconductor lasers with external optical feedback," Physical Review A: Atomic, Molecular and Optical Physics, vol. 58, no. 4, pp. R2672-R2675, 1998.

[16] F. Rogister, P. Mégret, O. Deparis, and M. Blondel, "Coexistence of in-phase and out-of-phase dynamics in a multimode external-cavity laser diode operating in the low-frequency fluctuations regime," Physical Review A: Atomic, Molecular and Optical Physics, vol. 62, no. 6, Article ID 061803, 4 pages, 2000.

[17] J. M. Buldú, J. García-Ojalvo, and M. C. Torrent, "Delayinduced resonances in an optical system with feedback," Physical Review E: Statistical, Nonlinear, and Soft Matter Physics, vol. 69, no. 4, Article ID 046207, 2004.

[18] S. K. Mandre, I. Fischer, and W. Elsäßer, "Spatiotemporal emission dynamics of a broad-area semiconductor laser in an external cavity: Stabilization and feedback-induced instabilities," Optics Communications, vol. 244, no. 1-6, pp. 355-365, 2005.

[19] S. Wolff, C. Doering, A. Rodionov, V. E. Sherstobitov, and H. Fouckhardt, "Self-pulsation in broad area lasers with transverse-mode selective feedback," Optics Communications, vol. 265, no. 2, pp. 642-648, 2006.

[20] D. Hoffmann, K. Huthmacher, C. Döring, and H. Fouckhardt, "Broad area lasers with monolithically integrated transverse mode selector," Applied Physics Letters, vol. 96, no. 18, Article ID 181104, 2010.

[21] D. Hoffmann, K. Huthmacher, C. Doering, and H. Fouckhardt, "Broad area lasers with folded-resonator geometry for integrated transverse mode selection," in Proceedings of the Novel In-Plane Semiconductor Lasers X, A. A. Belyanin and P. M. Smowton, Eds., vol. 7953, SPIE Photonics West, San Francisco, Calif, USA, 2011.

[22] K.-H. Hasler, B. Sumpf, P. Adamiec et al., "5-W DBR tapered lasers emitting at $1060 \mathrm{~nm}$ with a narrow spectral linewidth and a nearly diffraction-limited beam quality," IEEE Photonics Technology Letters, vol. 20, no. 19, pp. 1648-1650, 2008.

[23] C. Fiebig, G. Blume, C. Kaspari et al., "12 W high-brightness single-frequency DBR tapered diode laser," IEEE Electronics Letters, vol. 44, no. 21, pp. 1253-1255, 2008.

[24] M. Spreemann, M. Lichtner, M. Radziunas, U. Bandelow, and H. Wenzel, "Measurement and simulation of distributed-feedback tapered master-oscillator power amplifiers," IEEE Journal of Quantum Electronics, vol. 45, no. 6, pp. 609-616, 2009.

[25] I. Sergachev, R. Maulini, A. Bismuto, S. Blaser, T. Gresch, and A. Muller, "Gain-guided broad area quantum cascade lasers emitting $23.5 \mathrm{~W}$ peak power at room temperature," Optics Express, vol. 24, no. 17, pp. 19063-19071, 2016. 
[26] H. Fouckhardt, A.-K. Kleinschmidt, J. Strassner, and C. Doering, "Fundamental transverse mode selection (TMS\#0) of broad area semiconductor lasers with integrated twice-retracted $4 f$ set-up and film-waveguide lens," Advances in OptoElectronics, vol. 2017, Article ID 5283850, 6 pages, 2017.

[27] J. Salzman, R. Lang, A. Larson, and A. Yariv, "Confocal unstable-resonator semiconductor laser," Optics Expresss, vol.11, no. 8, pp. 507-509, 1986.

[28] A.-K. Kleinschmidt, "Resonatorinterne Transversalmodenselektion bei Antimonid-Breitstreifenlasern", ("Resonator-Internal Transverse Mode Selection of Antimonide Broad Area Lasers,") [Ph.D. thesis], Physics Department of the Technische Universität Kaiserslautern (TUK), Verlag Dr. Hut, Germany, 2017. 


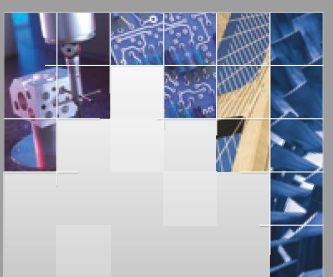

\section{Enfincering}
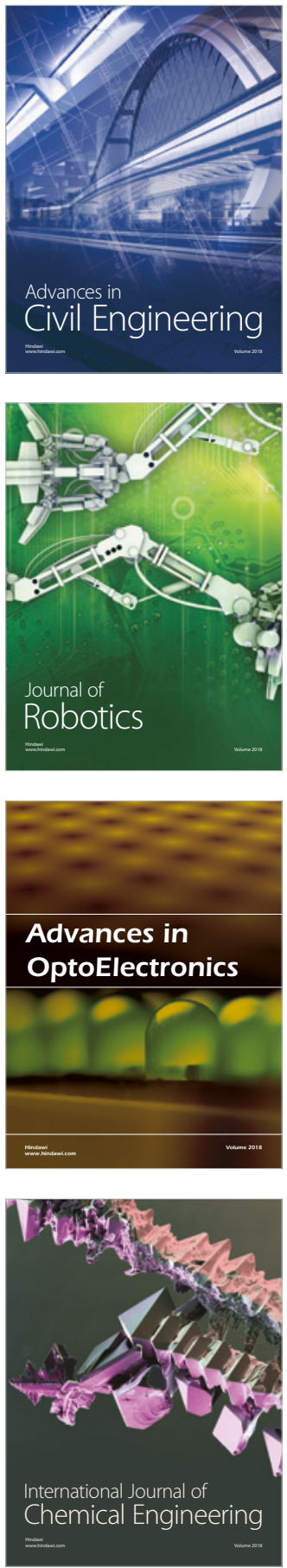

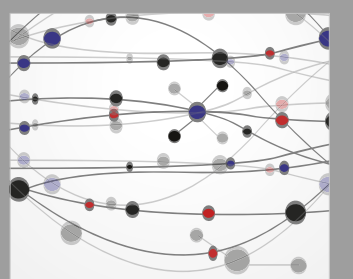

\section{Rotating \\ Machinery}

The Scientific World Journal

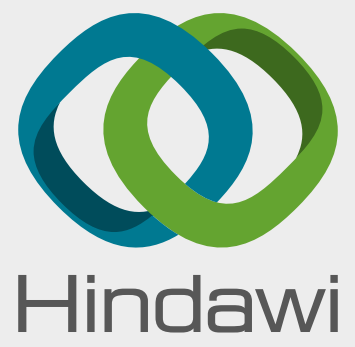

Submit your manuscripts at

www.hindawi.com
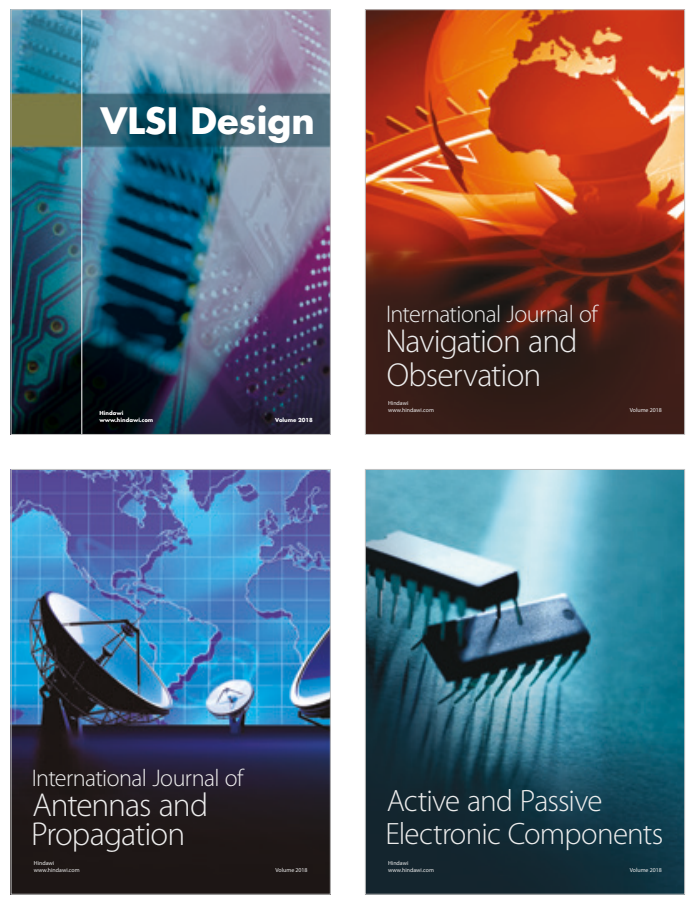
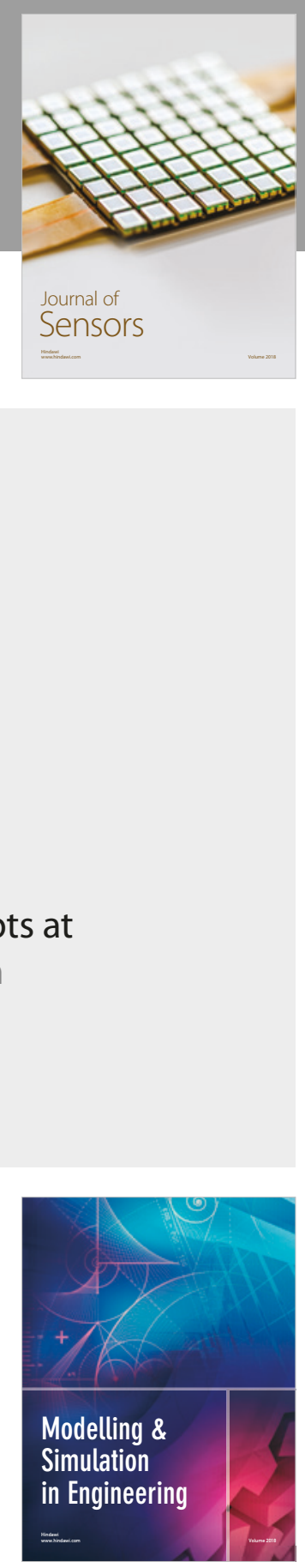

\section{Advances \\ Multimedia}
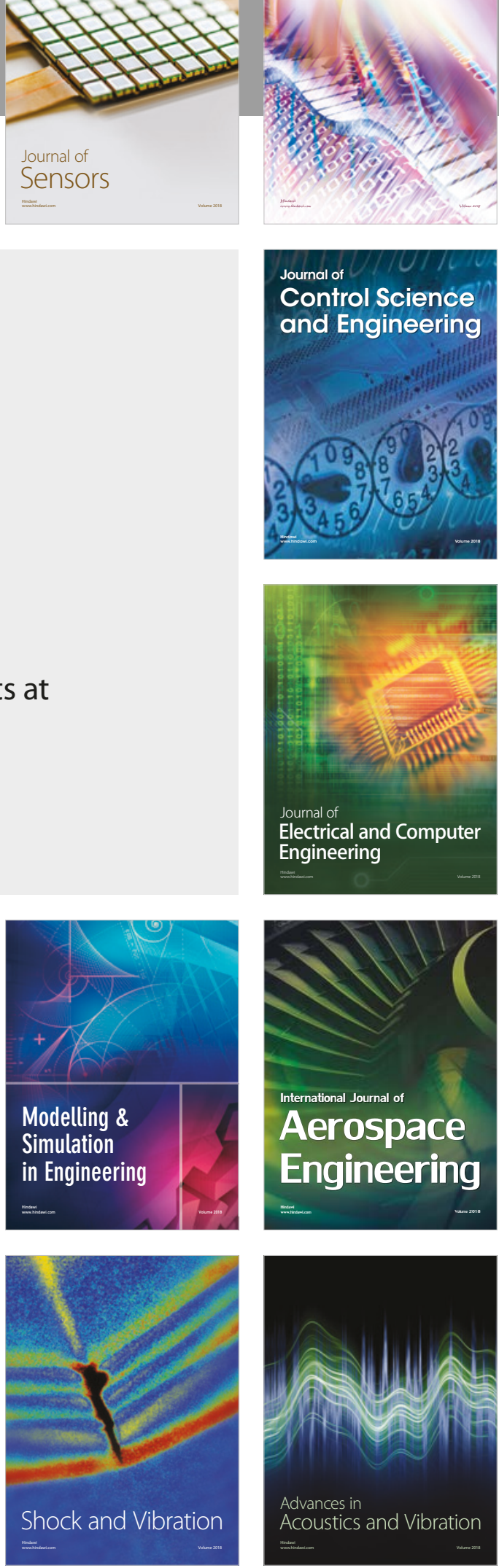